\title{
AN ASYMPTOTIC FORMULA FOR THE COEFFICIENTS OF $j(z)$
}

\author{
MICHAEL DEWAR* and M. RAM MURTY ${ }^{\dagger}$ \\ Department of Mathematics and Statistics \\ Queen's University, Kingston, ON, Canada K7L $3 N 6$ \\ *mdewar@mast.queensu.ca \\ ${ }^{\dagger}$ murty@mast.queensu.ca
}

Received 13 February 2012

Accepted 25 September 2012

Published

\begin{abstract}
We obtain a new proof of an asymptotic formula for the coefficients of the $j$-invariant of elliptic curves. Our proof does not use the circle method. We use Laplace's method of steepest descent and the Hardy-Ramanujan asymptotic formula for the partition function. (The latter asymptotic formula can be derived without the circle method.)

Keywords: Asymptotic formula; Fourier coefficients; $j$-invariant; weakly holomorphic modular forms.
\end{abstract}

Mathematics Subject Classification 2010: 11F30

\section{Introduction}

Suppose $k$ is an even integer and let $M_{k}$ denote the space of weight $k$ holomorphic modular forms on $\mathrm{SL}_{2}(\mathbb{Z})$. It is well known that the algebra of modular forms on $\mathrm{SL}_{2}(\mathbb{Z})$ is generated by Eisenstein series of the form

$$
E_{k}(\tau)=1-\frac{2 k}{B_{k}} \sum_{n=1}^{\infty} \sigma_{k-1}(n) q^{n},
$$

where $\sigma_{k-1}(n):=\sum_{d \mid n} d^{k-1}$ and $\frac{t}{e^{t}-1}=\sum_{k=0}^{\infty} B_{k} \frac{t^{k}}{k !}$ and $q=e^{2 \pi i z}$. The first non-trivial cusp form is

$$
\Delta(z)=q \prod_{n=1}^{\infty}\left(1-q^{n}\right)^{24}
$$

With this notation, $\Delta \in M_{12}$ and $E_{k} \in M_{k}$ whenever $k \geq 4$ is even.

The modular function

$$
j(z)=\frac{E_{12}(z)}{\Delta(z)}+\frac{432000}{691}=q^{-1}+744+\sum_{n=1}^{\infty} c(n) q^{n}
$$


is a fundamental object in number theory. Petersson [7], and later Rademacher [8] independently, used the circle method to prove the asymptotic formula

$$
c(n) \sim \frac{e^{4 \pi \sqrt{n}}}{\sqrt{2} n^{3 / 4}},
$$

as $n \rightarrow \infty$.

Petersson and Rademacher were inspired by the seminal work of Hardy and Ramanujan [4] which introduced the circle method in order to prove the asymptotic formula for the partition function

$$
p(n) \sim \frac{e^{\pi \sqrt{\frac{2 n}{3}}}}{4 \sqrt{3} n},
$$

as $n \rightarrow \infty$, where $p(n)$ is determined by

$$
\sum_{n=0}^{\infty} p(n) q^{n}=\prod_{n=1}^{\infty}\left(1-q^{n}\right)^{-1}
$$

In a recent article [2] we gave a new proof of (1.2) without using the circle method. Our derivation of (1.2) used an algebraic formula of Bruinier and Ono [1].

The purpose of the present article is to give a new proof of (1.1) without using the circle method. We use Laplace's method of steepest descent and the HardyRamanujan asymptotic. In fact, our method yields much more general conclusions.

Let $M_{k}^{!}$denote the space of weight $k$ weakly holomorphic modular forms on $\mathrm{SL}_{2}(\mathbb{Z})$. That is, meromorphic modular forms whose only poles (if any) are at $i \infty$. If $f \in M_{k}^{!}$has $\operatorname{ord}_{i \infty} f=-m<0$, then

$$
f=\sum_{j=0}^{\lfloor k / 12\rfloor+m} b_{j} E_{k+12(m-j)} \Delta^{j-m},
$$

for some $b_{j} \in \mathbb{C}$ where $b_{0} \neq 0$. We have the following theorem which immediately implies (1.1).

Theorem 1. Suppose $k \in 2 \mathbb{Z}$ and $f \in M_{k}^{!}$has $\operatorname{ord}_{i \infty} f=-m<0$ and $f=$ $\sum_{n=-m}^{\infty} \lambda_{f}(n) q^{n}$. Then

$$
\lambda_{f}(n) \sim i^{k} \frac{\lambda_{f}(-m)}{\sqrt{2 n}}\left(\frac{n}{m}\right)^{\frac{k}{2}-\frac{1}{4}} e^{4 \pi \sqrt{n m}} .
$$

Our proof of Theorem 1 has two main steps. The first - which may be of independent interest - uses Laplace's method to prove the following.

Theorem 2. Suppose

$$
\begin{aligned}
& f(z)=\sum_{n=0}^{\infty} \lambda_{f}(n) q^{n}, \\
& g(z)=\sum_{n=0}^{\infty} \lambda_{g}(n) q^{n},
\end{aligned}
$$


1

where

$$
\begin{aligned}
& \lambda_{f}(n) \sim c_{f} n^{\alpha} e^{A \sqrt{n}}, \\
& \lambda_{g}(n) \sim c_{g} n^{\beta} e^{B \sqrt{n}},
\end{aligned}
$$

with $\alpha, \beta, A, B, c_{f}, c_{g} \in \mathbb{R}$ and $A, B, c_{f}, c_{g}>0$. Then for $f g(z)=\sum_{n=0}^{\infty} \lambda_{f g}(n) q^{n}$ we have

$$
\lambda_{f g}(n) \sim c_{f} c_{g} 2 \sqrt{2 \pi} \frac{A^{2 \alpha+1} B^{2 \beta+1}}{\left(A^{2}+B^{2}\right)^{\frac{5}{4}+\alpha+\beta}} n^{\alpha+\beta+\frac{3}{4}} e^{\sqrt{A^{2}+B^{2}} \sqrt{n}} .
$$

Here is the strategy to prove Theorem 1 . Set

$$
\sum_{n=0}^{\infty} p^{(j)}(n) q^{n}=\prod_{n=0}^{\infty}\left(1-q^{n}\right)^{-j}
$$

By Theorem 2 and (1.2), an easy induction shows that $p^{(j)}(n) \sim c_{j} n^{\alpha_{j}} e^{A_{j} \sqrt{n}}$ where

$$
\begin{aligned}
c_{j} & =\frac{1}{\sqrt{2}}\left(\frac{j}{24}\right)^{\frac{j+1}{4}}, \\
\alpha_{j} & =-\frac{j}{4}-\frac{3}{4}, \\
A_{j} & =\pi \sqrt{\frac{2 j}{3}} .
\end{aligned}
$$

Thus for any fixed integer $m>0$, the coefficients of

$$
q^{m} \Delta^{-m}=\prod_{n=1}^{\infty}\left(1-q^{n}\right)^{-24 m}=\sum_{n=0}^{\infty} p^{(24 m)}(n) q^{n}
$$

satisfy the asymptotic formula

$$
p^{(24 m)}(n) \sim \frac{1}{\sqrt{2 n}}\left(\frac{m}{n}\right)^{6 m+\frac{1}{4}} e^{4 \pi \sqrt{n m}} .
$$

In light of (1.3), we prove the following.

Theorem 3. Suppose $k \geq 4$ is even and

$$
f(z)=\sum_{n=0}^{\infty} \lambda_{f}(n) q^{n},
$$

where $\lambda_{f}(n) \geq 0$ for all $n$ and

$$
\lambda_{f}(n) \sim \frac{c_{f} e^{A \sqrt{n}}}{n^{\alpha}},
$$

with real numbers $c_{f}, A, \alpha>0$. Then for $f E_{k}(z)=\sum_{n=0}^{\infty} \lambda_{f E_{k}}(n) q^{n}$ we have

$$
\lambda_{f E_{k}}(n) \sim \frac{c_{f} e^{A \sqrt{n}}}{n^{\alpha-\frac{k}{2}}}\left(\frac{4 \pi i}{A}\right)^{k} .
$$


The proof of Theorem 1 follows easily from Theorems 2 and 3 .

Proof of Theorem 1. Let $\lambda_{E_{k+12(m-j)} \Delta^{j-m}}(n)$ denote the $n$th coefficient of $E_{k+12(m-j)} \Delta^{j-m}$. For fixed $m$ and $j$, we obviously have

$$
\lambda_{E_{k+12(m-j)} \Delta^{j-m}}(n) \sim \lambda_{E_{k+12(m-j)} \Delta^{j-m}}(n+j-m)=\lambda_{E_{k+12(m-j)} q^{m-j} \Delta^{j-m}}(n) .
$$

Hence by (1.3), Theorem 3 and (1.5), we have

$$
\begin{aligned}
\lambda_{f}(n) & =\sum_{j=0}^{\lfloor k / 12\rfloor+m} b_{j} \lambda_{E_{k+12(m-j)} \Delta^{j-m}}(n) \\
& \sim \sum_{j=0}^{m-1} b_{j} \frac{i^{k}}{\sqrt{2 n}}\left(\frac{n}{m-j}\right)^{\frac{k}{2}-\frac{1}{4}} e^{4 \pi \sqrt{n(m-j)}}+O\left(n^{k-1}\right) \\
& \sim b_{0} \frac{i^{k}}{\sqrt{2 n}}\left(\frac{n}{m}\right)^{\frac{k}{2}-\frac{1}{4}} e^{4 \pi \sqrt{n m}},
\end{aligned}
$$

since the $e^{4 \pi \sqrt{n m}}$ term dominates all of the other exponentials. Finally, observe that $b_{0}=\lambda_{f}(-m)$.

\section{Proof of Theorem 2}

The key to proving Theorem 2 is that $\lambda_{f g}(n)=\sum_{j} \lambda_{f}(j) \lambda_{g}(n-j)$ is approximated by

$$
c_{f} c_{g} \sum_{j} j^{\alpha}(n-j)^{\beta} e^{A \sqrt{j}+B \sqrt{n-j}}=c_{f} c_{g} n^{\alpha+\beta} \sum_{j} G\left(\frac{j}{n}\right) e^{\sqrt{n} F\left(\frac{j}{n}\right)},
$$

where

$$
\begin{aligned}
& F(x):=A \sqrt{x}+B \sqrt{1-x}:(0,1) \rightarrow \mathbb{R}_{>0}, \\
& G(x):=x^{\alpha}(1-x)^{\beta}:(0,1) \rightarrow \mathbb{R}_{>0} .
\end{aligned}
$$

Set

$$
c:=\frac{A^{2}}{A^{2}+B^{2}} .
$$

The function $F(x)$ is increasing on $(0, c)$, has a maximum of $\sqrt{A^{2}+B^{2}}$ at $x=c$, and is decreasing on $(c, 1)$.

Proof of Theorem 2. Let $0<\epsilon<1$ be given. By continuity, there exists $\delta>0$ such that if $|x-c|<2 \delta$, then

$$
(1-\epsilon) G(c)<G(x)<(1+\epsilon) G(c) .
$$

We may assume that both $\delta<c$ and $\delta<1-c$. By (1.4), for large enough $n$,

$$
\begin{aligned}
& (1-\epsilon) c_{f} n^{\alpha} e^{A \sqrt{n}}<\lambda_{f}(n)<(1+\epsilon) c_{f} n^{\alpha} e^{A \sqrt{n}}, \\
& (1-\epsilon) c_{g} n^{\beta} e^{B \sqrt{n}}<\lambda_{g}(n)<(1+\epsilon) c_{g} n^{\beta} e^{B \sqrt{n}} .
\end{aligned}
$$


Decompose

$$
\begin{aligned}
\lambda_{f g}(n)= & \underbrace{\sum_{j=0}^{\lceil(c-\delta) n\rceil-1} \lambda_{f}(j) \lambda_{g}(n-j)}_{=: S_{0}(n)}+\underbrace{\sum_{j=\lceil(c-\delta) n\rceil}^{\lfloor(c+\delta) n\rfloor} \lambda_{f}(j) \lambda_{g}(n-j)}_{=: S_{1}(n)} \\
& +\underbrace{\sum_{j=\lfloor(c+\delta) n\rfloor+1}^{n} \lambda_{f}(j) \lambda_{g}(n-j)}_{=: S_{2}(n)} .
\end{aligned}
$$

By (1.4),

$$
\begin{aligned}
S_{0}(n) & =O\left(n^{|\alpha|+|\beta|} \sum_{j=0}^{\lceil(c-\delta) n\rceil-1} e^{\sqrt{n} F\left(\frac{j}{n}\right)}\right)=O\left(n^{|\alpha|+|\beta|+1} e^{\sqrt{n} F(c-\delta)}\right) \\
& =o\left(n^{\alpha+\beta+3 / 4} e^{\sqrt{n} F(c)}\right) .
\end{aligned}
$$

Similarly,

$$
S_{2}(n)=O\left(n^{|\alpha|+|\beta|+1} e^{\sqrt{n} F(c+\delta)}\right)=o\left(n^{\alpha+\beta+3 / 4} e^{\sqrt{n} F(c)}\right) .
$$

It remains to consider $S_{1}(n)$. For large enough $n$, the inequalities $(2.2)$ apply to every summand in $S_{1}(n)$ :

$$
\begin{aligned}
& (1-\epsilon)^{2} c_{f} c_{g} n^{\alpha+\beta} \sum_{j=\lceil(c-\delta) n\rceil}^{\lfloor(c+\delta) n\rfloor} G\left(\frac{j}{n}\right) e^{\sqrt{n} F\left(\frac{j}{n}\right)} \\
& \quad<S_{1}(n)<(1+\epsilon)^{2} c_{f} c_{g} n^{\alpha+\beta} \sum_{j=\lceil(c-\delta) n\rceil}^{\lfloor(c+\delta) n\rfloor} G\left(\frac{j}{n}\right) e^{\sqrt{n} F\left(\frac{j}{n}\right)} .
\end{aligned}
$$

By (2.1), we have

$$
\begin{aligned}
& (1-\epsilon)^{3} c_{f} c_{g} G(c) n^{\alpha+\beta+1} \sum_{j=\lceil(c-\delta) n\rceil}^{\lfloor(c+\delta) n\rfloor} e^{\sqrt{n} F\left(\frac{j}{n}\right)} \cdot \frac{1}{n} \\
& \quad<S_{1}(n)<(1+\epsilon)^{3} c_{f} c_{g} G(c) n^{\alpha+\beta+1} \sum_{j=\lceil(c-\delta) n\rceil}^{\lfloor(c+\delta) n\rfloor} e^{\sqrt{n} F\left(\frac{j}{n}\right)} \cdot \frac{1}{n} .
\end{aligned}
$$

We now consider

$$
\widehat{S}_{1}(n):=\sum_{j=\lceil(c-\delta) n\rceil}^{\lfloor(c+\delta) n\rfloor} e^{\sqrt{n} F\left(\frac{j}{n}\right)} \cdot \frac{1}{n}
$$

and compare it to the integral

$$
I(n):=\int_{c-\delta}^{c+\delta} e^{\sqrt{n} F(x)} d x .
$$


Lemma 4. Let $\widehat{S}_{1}(n)$ and $I(n)$ be as above. Then

$$
I(n)-\frac{e^{\sqrt{n} F(c)}}{n} \leq \widehat{S}_{1}(n) \leq I(n)+\frac{e^{\sqrt{n} F(c)}}{n} .
$$

Proof. First observe that

$$
I(n) \leq \int_{\frac{\lfloor(c-\delta) n\rceil}{n}-\frac{1}{n}}^{\frac{\lfloor(c+\delta) n\rfloor}{n}+\frac{1}{n}} e^{\sqrt{n} F(x)} d x=: I_{\mathrm{over}}(n) .
$$

Recall that right-end-point Riemann sums overestimate integrals of increasing functions and left-end-point Riemann sums overestimate integrals of decreasing functions. Thus we obtain an upper bound for $I_{\text {over }}(n)$ by constructing a Riemann sum (of rectangles of width $1 / n$ ) with right end-points for the interval to the left of $c$ and with left end-points for the interval to the right of $c$. In particular

$$
I(n) \leq I_{\text {over }}(n) \leq \widehat{S}_{1}(n)+\frac{e^{\sqrt{n} F(c)}}{n},
$$

where the $\frac{e^{\sqrt{n} F(c)}}{n}$ term must be added to cover the gap between the right-end-point rectangles and the left-end-point rectangles.

Similarly, we observe that

$$
I(n) \geq \int_{\frac{\lfloor(c-\delta) n]}{n}}^{\frac{\lfloor(c+\delta) n\rfloor}{n}} e^{\sqrt{n} F(x)} d x=: I_{\text {under }}(n) .
$$

An underestimate for $I_{\text {under }}$ is obtained by constructing a Riemann sum with left end-points for the interval to the left of $c$ and right end-points to the right of $c$. In particular,

$$
I(n) \geq I_{\text {under }}(n) \geq \widehat{S}_{1}(n)-\frac{e^{\sqrt{n} F(c)}}{n},
$$

where the $-\frac{e^{\sqrt{n} F(c)}}{n}$ term compensates for the overlap between the left-end-point rectangles and the right-end-point rectangles. The lemma follows immediately.

Laplace's method (see, for example, [3, Chap. 19.3]) implies that

$$
I(n) \sim \sqrt{\frac{2 \pi}{\sqrt{n}\left|F^{\prime \prime}(c)\right|}} \cdot e^{\sqrt{n} F(c)} .
$$

That is, for large enough $n$ we have

$$
(1-\epsilon) \sqrt{\frac{2 \pi}{\left|F^{\prime \prime}(c)\right|}} n^{-1 / 4} e^{\sqrt{n} F(c)}<I(n)<(1+\epsilon) \sqrt{\frac{2 \pi}{\left|F^{\prime \prime}(c)\right|}} n^{-1 / 4} e^{\sqrt{n} F(c)} .
$$


Combining this with (2.3) and Lemma 4 gives

$$
\begin{aligned}
S_{1}(n) & <(1+\epsilon)^{3} c_{f} c_{g} G(c) n^{\alpha+\beta+1}\left\{I(n)+\frac{e^{\sqrt{n} F(c)}}{n}\right\} \\
& <(1+\epsilon)^{3} c_{f} c_{g} G(c) n^{\alpha+\beta+1}\left\{(1+\epsilon) \sqrt{\frac{2 \pi}{\left|F^{\prime \prime}(c)\right|}} n^{-1 / 4} e^{\sqrt{n} F(c)}+\frac{e^{\sqrt{n} F(c)}}{n}\right\} \\
& =(1+\epsilon)^{4} c_{f} c_{g} G(c) \sqrt{\frac{2 \pi}{\left|F^{\prime \prime}(c)\right|}} n^{\alpha+\beta+\frac{3}{4}} e^{\sqrt{n} F(c)}+o\left(n^{\alpha+\beta+\frac{3}{4}} e^{\sqrt{n} F(c)}\right)
\end{aligned}
$$

and similarly

$$
S_{1}(n)>(1-\epsilon)^{4} c_{f} c_{g} G(c) \sqrt{\frac{2 \pi}{\left|F^{\prime \prime}(c)\right|}} n^{\alpha+\beta+\frac{3}{4}} e^{\sqrt{n} F(c)}+o\left(n^{\alpha+\beta+\frac{3}{4}} e^{\sqrt{n} F(c)}\right) .
$$

We conclude that

$$
S(n) \sim S_{1}(n) \sim c_{f} c_{g} G(c) \sqrt{\frac{2 \pi}{\left|F^{\prime \prime}(c)\right|}} n^{\alpha+\beta+\frac{3}{4}} e^{\sqrt{n} F(c)} .
$$

Finally, it is elementary that $F(c)=\sqrt{A^{2}+B^{2}}$, that $\left|F^{\prime \prime}(c)\right|=\frac{\left(A^{2}+B^{2}\right)^{5 / 2}}{4 A^{2} B^{2}}$, and that $G(c)=\frac{A^{2 \alpha} B^{2 \beta}}{\left(A^{2}+B^{2}\right)^{\alpha+\beta}}$.

\section{Proof of Theorem 3}

It is convenient to set $g=i^{k}\left(E_{k}-1\right)=\sum_{n=1}^{\infty} \lambda_{g}(n) q^{n}$ so that $\lambda_{g}(n) \geq 0$. We will show that for $f g=\sum_{n=0}^{\infty} \lambda_{f g}(n) q^{n}$ we have

$$
\lambda_{f g}(n) \sim \frac{c_{f} e^{A \sqrt{n}}}{n^{\alpha-\frac{k}{2}}}\left(\frac{4 \pi}{A}\right)^{k} .
$$

Once (3.1) is established, it is easy to see that $f E_{k}=f+i^{k} f g$ and so

$$
\begin{aligned}
\lambda_{f E_{k}}(n) & \sim \lambda_{f}(n)+i^{k} \lambda_{f g}(n) \\
& \sim \frac{c_{f} e^{A \sqrt{n}}}{n^{\alpha}}+i^{k} \frac{c_{f} e^{A \sqrt{n}}}{n^{\alpha-\frac{k}{2}}}\left(\frac{4 \pi}{A}\right)^{k} \\
& \sim \frac{c_{f} e^{A \sqrt{n}}}{n^{\alpha-\frac{k}{2}}}\left(\frac{4 \pi i}{A}\right)^{k},
\end{aligned}
$$

as desired. We now prove (3.1).

Once again, the key observation is that $\lambda_{f g}(n)=\sum_{j} \lambda_{f}(n-j) \lambda_{g}(j)$ is approximated by

$$
\frac{c_{f}}{n^{\alpha}} \sum_{j} G\left(\frac{j}{n}\right) e^{A \sqrt{n} F\left(\frac{j}{n}\right)} \lambda_{g}(j)
$$


where

$$
\begin{aligned}
& F(x):=\sqrt{1-x}:(0,1) \rightarrow \mathbb{R}_{>0}, \\
& G(x):=\frac{1}{(1-x)^{\alpha}}:(0,1) \rightarrow \mathbb{R}_{>0} .
\end{aligned}
$$

Elementary calculus (Taylor's theorem) gives that for $0 \leq x \leq \delta<1$,

$$
\begin{aligned}
1-\frac{x}{2}-\frac{\delta^{2}}{8(1-\delta)^{3 / 2}} & \leq F(x) \leq 1-\frac{x}{2} \\
1 & \leq G(x) \leq 1+\alpha \frac{\delta}{(1-\delta)^{\alpha+1}} .
\end{aligned}
$$

Since $e^{A \sqrt{n} x}$ is an increasing function of $x,(3.2)$ implies

$$
e^{A \sqrt{n}\left\{1-\frac{x}{2}-\frac{\delta^{2}}{8(1-\delta)^{3 / 2}}\right\}} \leq e^{A \sqrt{n} F(x)} \leq e^{A \sqrt{n}\left\{1-\frac{x}{2}\right\}}
$$

for all $0 \leq x \leq \delta<1$. In particular, for integers $0 \leq j \leq \delta n$, take $x=j / n$ above to deduce

$$
e^{A \sqrt{n}-\frac{A j}{2 \sqrt{n}}-\frac{A \sqrt{n} \delta^{2}}{8(1-\delta)^{3 / 2}}} \leq e^{A \sqrt{n-j}} \leq e^{A \sqrt{n}-\frac{A j}{2 \sqrt{n}}} .
$$

We will establish (3.1) in two steps. We first show that

$$
\limsup _{n \rightarrow \infty} \frac{\lambda_{f g}(n)}{c_{f}\left(\frac{4 \pi}{A}\right)^{k} \frac{e^{A \sqrt{n}}}{n^{\alpha-k / 2}}} \leq 1
$$

Let $\epsilon>0$ be given. By continuity, we can fix $0<\delta<1$ such that for $0 \leq x \leq \delta$ we have

$$
G(x) \leq(1+\epsilon) .
$$

By assumption, $\lambda_{f}(n)=O\left(e^{A \sqrt{n}}\right)$ and $\lambda_{g}(n)=O\left(n^{k-1}\right)$. Hence $\lambda_{f}(n-j) \lambda_{g}(j)=$ $O\left(e^{A \sqrt{n-j}} j^{k-1}\right)$ and so

$$
\sum_{j=\lfloor\delta n\rfloor+1}^{n} \lambda_{f}(n-j) \lambda_{g}(j)=O\left(e^{A \sqrt{n-n \delta}} n^{k}\right)=O\left(e^{A \sqrt{1-\delta} \sqrt{n}} n^{k}\right)=o\left(\frac{e^{A \sqrt{n}}}{n^{\alpha-k / 2}}\right) .
$$

Thus, it suffices to consider

$$
S_{\delta}(n):=\sum_{j=0}^{\lfloor\delta n\rfloor} \lambda_{f}(n-j) \lambda_{g}(j)
$$

By (1.6), for large enough $n, \lambda_{f}(n)<(1+\epsilon) c_{f} e^{A \sqrt{n}} / n^{\alpha}$. Thus for large enough $n$ and $j \leq \delta n$,

$$
\lambda_{f}(n-j)<(1+\epsilon) c_{f} \frac{e^{A \sqrt{n-j}}}{(n-j)^{\alpha}} .
$$


Thus by (3.4) and (3.6), this implies that for large $n$

$$
\begin{aligned}
S_{\delta}(n) & <(1+\epsilon) \frac{c_{f}}{n^{\alpha}} \sum_{j=0}^{\lfloor\delta n\rfloor} G\left(\frac{j}{n}\right) e^{A \sqrt{n-j}} \lambda_{g}(j) \\
& <(1+\epsilon)^{2} c_{f} \frac{e^{A \sqrt{n}}}{n^{\alpha}} \sum_{j=0}^{\lfloor\delta n\rfloor} \lambda_{g}(j) e^{-\frac{A j}{2 \sqrt{n}}} .
\end{aligned}
$$

By the non-negativity of all of the terms,

$$
\begin{aligned}
S_{\delta}(n) & <(1+\epsilon)^{2} c_{f} \frac{e^{A \sqrt{n}}}{n^{\alpha}} \sum_{j=0}^{\infty} \lambda_{g}(j) e^{-\frac{A j}{2 \sqrt{n}}} \\
& =(1+\epsilon)^{2} c_{f} \frac{e^{A \sqrt{n}}}{n^{\alpha}} g\left(\frac{A i}{4 \pi \sqrt{n}}\right) .
\end{aligned}
$$

Since $g=i^{k} E_{k}-i^{k}$, the modularity of $E_{k}$ implies

$$
g\left(\frac{A i}{4 \pi \sqrt{n}}\right)=\left(\frac{4 \pi \sqrt{n}}{A}\right)^{k} E_{k}\left(\frac{4 \pi \sqrt{n} i}{A}\right)-i^{k} .
$$

Since $\lim _{n \rightarrow \infty} E_{k}\left(\frac{4 \pi \sqrt{n} i}{A}\right)=1$, for large enough $n$ we have

$$
g\left(\frac{A i}{4 \pi \sqrt{n}}\right)<(1+\epsilon)\left(\frac{4 \pi \sqrt{n}}{A}\right)^{k} .
$$

Combining this with (3.7) shows that for large enough $n$,

$$
S_{\delta}(n)<(1+\epsilon)^{3} c_{f} \frac{e^{A \sqrt{n}}}{n^{\alpha-k / 2}}\left(\frac{4 \pi}{A}\right)^{k} .
$$

Since $\epsilon>0$ was arbitrary, (3.5) follows immediately.

It remains to show that

$$
\liminf _{n \rightarrow \infty} \frac{\lambda_{f g}(n)}{c_{f}\left(\frac{4 \pi}{A}\right)^{k} \frac{e^{A \sqrt{n}}}{n^{\alpha-k / 2}}} \geq 1 .
$$

Let $\epsilon>0$ be given and set $\delta=n^{-1 / 3}$. By the non-negativity of the terms,

$$
\lambda_{f g}(n) \geq \sum_{j=0}^{\lfloor\delta n\rfloor} \lambda_{f}(n-j) \lambda_{g}(j)=S_{\delta}(n) .
$$

By (1.6), for large enough $n$,

$$
S_{\delta}(n)>(1-\epsilon) \frac{c_{f}}{n^{\alpha}} \sum_{j=0}^{\lfloor\delta n\rfloor} G\left(\frac{j}{n}\right) e^{A \sqrt{n-j}} \lambda_{g}(j) .
$$


1 By (3.3) and (3.4), for large enough $n$,

$$
S_{\delta}(n)>(1-\epsilon) e^{-\frac{A \sqrt{n} \delta^{2}}{8(1-\delta)^{3 / 2}}} c_{f} \frac{e^{A \sqrt{n}}}{n^{\alpha}} \sum_{j=0}^{\lfloor\delta n\rfloor} e^{-\frac{A j}{2 \sqrt{n}}} \lambda_{g}(j) .
$$

3 In a moment we will prove the following.

Lemma 5. For $\delta=n^{-1 / 3}$, we have

$$
\lim _{n \rightarrow \infty} \frac{\sum_{j=\lfloor\delta n\rfloor+1}^{\infty} \lambda_{g}(j) e^{-\frac{A j}{2 \sqrt{n}}}}{\sum_{j=0}^{\infty} \lambda_{g}(j) e^{-\frac{A j}{2 \sqrt{n}}}}=0
$$

Since obviously

$$
\sum_{j=0}^{\lfloor\delta n\rfloor} \lambda_{g}(j) e^{-\frac{A j}{2 \sqrt{n}}}=g\left(\frac{A i}{4 \pi \sqrt{n}}\right)\left(1-\frac{\sum_{j=\lfloor\delta n\rfloor+1}^{\infty} \lambda_{g}(j) e^{-\frac{A j}{2 \sqrt{n}}}}{\sum_{j=0}^{\infty} \lambda_{g}(j) e^{-\frac{A j}{2 \sqrt{n}}}}\right)
$$

for large enough $n$ Lemma 5 implies

$$
S_{\delta}(n)>(1-\epsilon)^{2} e^{-\frac{A \sqrt{n} \delta^{2}}{8(1-\delta)^{3 / 2}}} c_{f} \frac{e^{A \sqrt{n}}}{n^{\alpha}} g\left(\frac{A i}{4 \pi \sqrt{n}}\right) .
$$

Since $g=i^{k} E_{k}-i^{k}$, the modularity of $E_{k}$ implies

$$
g\left(\frac{A i}{4 \pi \sqrt{n}}\right)=\left(\frac{4 \pi \sqrt{n}}{A}\right)^{k} E_{k}\left(\frac{4 \pi \sqrt{n} i}{A}\right)-i^{k} .
$$

Since $\lim _{n \rightarrow \infty} E_{k}\left(\frac{4 \pi \sqrt{n} i}{A}\right)=1$, for large enough $n$ we have

$$
g\left(\frac{A i}{4 \pi \sqrt{n}}\right)>(1-\epsilon)\left(\frac{4 \pi \sqrt{n}}{A}\right)^{k} .
$$

Combining this with (3.9) shows that for large enough $n$,

$$
S_{\delta}(n)>(1-\epsilon)^{3} e^{-\frac{A \sqrt{n} \delta^{2}}{8(1-\delta)^{3 / 2}}} c_{f}\left(\frac{4 \pi}{A}\right)^{k} \frac{e^{A \sqrt{n}}}{n^{\alpha-k / 2}} .
$$

Since $\lim _{n \rightarrow \infty} \frac{\sqrt{n} \delta^{2}}{(1-\delta)^{3 / 2}}=\lim _{n \rightarrow \infty} \frac{n^{-1 / 6}}{\left(1-n^{-1 / 3}\right)^{3 / 2}}=0$, the inequality (3.8) follows immediately. It remains to prove the lemma.

Proof of Lemma 5. We first claim that for all integers $j \geq 1$ and all real $0<\beta<1$,

$$
\sigma_{k-1}(j) \leq j^{k} \leq\left(\frac{k}{\beta}\right)^{k} e^{\beta j}
$$


1 On the one hand, if $j \leq k / \beta$, then $j^{k} \leq(k / \beta)^{k} \leq(k / \beta)^{k} e^{\beta j}$. On the other hand, if $2 j>k / \beta$, then

$$
j^{k} \leq\left(\frac{k}{\beta}\right)^{k} e^{\beta j} \Leftrightarrow\left(\frac{\beta}{k}\right)^{k} \leq \frac{e^{\beta j}}{j^{k}} .
$$

$4 \quad$ Since the function $e^{\beta x} / x^{k}$ is non-decreasing for $x \geq k / \beta$, we know

$$
\frac{e^{\beta j}}{j^{k}} \geq \frac{e^{\beta\left(\frac{k}{\beta}\right)}}{\left(\frac{k}{\beta}\right)^{k}}=\left(\frac{\beta}{k}\right)^{k} e^{k}>\left(\frac{\beta}{k}\right)^{k}
$$

6 This proves the claim.

Hence, for all $0<\beta<1$,

$$
\begin{aligned}
0<\frac{\sum_{j=\lfloor\delta n\rfloor+1}^{\infty} \lambda_{g}(j) e^{-\frac{A j}{2 \sqrt{n}}}}{\sum_{j=0}^{\infty} \lambda_{g}(j) e^{-\frac{A j}{2 \sqrt{n}}}} \leq \frac{\sum_{j=\lfloor\delta n\rfloor+1}^{\infty} \sigma_{k-1}(j) e^{-\frac{A j}{2 \sqrt{n}}}}{\sum_{j=1}^{\infty} e^{-\frac{A j}{2 \sqrt{n}}}} \\
\leq\left(\frac{k}{\beta}\right)^{k} \frac{\sum_{j=\lfloor\delta n\rfloor+1}^{\infty} e^{\left(\beta-\frac{A}{2 \sqrt{n}}\right) j}}{\sum_{j=1}^{\infty} e^{-\frac{A j}{2 \sqrt{n}}}}
\end{aligned}
$$

If $\beta-\frac{A}{2 \sqrt{n}}<0$, then the geometric series converge to give

$$
\begin{aligned}
\frac{\sum_{j=\lfloor\delta n\rfloor+1}^{\infty} \lambda_{g}(j) e^{-\frac{A j}{2 \sqrt{n}}}}{\sum_{j=0}^{\infty} \lambda_{g}(j) e^{-\frac{A j}{2 \sqrt{n}}}} & \leq\left(\frac{k}{\beta}\right)^{k}\left(\frac{1-e^{-\frac{A}{2 \sqrt{n}}}}{e^{-\frac{A}{2 \sqrt{n}}}}\right)\left(\frac{e^{\left(\beta-\frac{A}{2 \sqrt{n}}\right)(\lfloor\delta n\rfloor+1)}}{1-e^{\left(\beta-\frac{A}{2 \sqrt{n}}\right)}}\right) \\
& \leq\left(\frac{k}{\beta}\right)^{k}\left(\frac{1-e^{-\frac{A}{2 \sqrt{n}}}}{e^{-\frac{A}{2 \sqrt{n}}}}\right)\left(\frac{e^{\left(\beta-\frac{A}{2 \sqrt{n}}\right) \delta n}}{1-e^{\left(\beta-\frac{A}{2 \sqrt{n}}\right)}}\right)
\end{aligned}
$$

$7 \quad$ Choose $\beta=\frac{A}{2 n^{3 / 2}}$. Now

8

$$
\frac{\sum_{j=\lfloor\delta n\rfloor+1}^{\infty} \lambda_{g}(j) e^{-\frac{A j}{2 \sqrt{n}}}}{\sum_{j=0}^{\infty} \lambda_{g}(j) e^{-\frac{A j}{2 \sqrt{n}}}} \leq\left(\frac{2 k}{A}\right)^{k}\left(\frac{1-e^{-\frac{A}{2 \sqrt{n}}}}{1-e^{-\frac{A}{2 \sqrt{n}}\left(1-\frac{1}{n}\right)}}\right)\left(\frac{n^{3 k / 2}}{e^{\frac{A \delta \sqrt{n}}{2}\left(1-\frac{1}{n}\right)}}\right) e^{\frac{A}{2 \sqrt{n}}}
$$


Substitute $\delta=n^{-1 / 3}$ to get

$$
\begin{aligned}
\frac{\sum_{j=\lfloor\delta n\rfloor+1}^{\infty} \lambda_{g}(j) e^{-\frac{A j}{2 \sqrt{n}}}}{\sum_{j=0}^{\infty} \lambda_{g}(j) e^{-\frac{A j}{2 \sqrt{n}}}} & \leq\left(\frac{2 k}{A}\right)^{k}\left(\frac{1-e^{-\frac{A}{2 \sqrt{n}}}}{1-e^{-\frac{A}{2 \sqrt{n}}\left(1-\frac{1}{n}\right)}}\right)\left(\frac{n^{3 k / 2}}{e^{\frac{A n^{1 / 6}}{2}\left(1-\frac{1}{n}\right)}}\right) e^{-\frac{A}{2 \sqrt{n}}} \\
& \rightarrow\left(\frac{2 k}{A}\right)^{k} \cdot 1 \cdot 0 \cdot 1,
\end{aligned}
$$

as $n \rightarrow \infty$. This proves the lemma.

\section{Acknowledgments}

The authors would like to thank the referee for various interesting remarks: Theorem 1 should also follow from the development of Poincare series (see [6]). There will of course be problems with convergence for low weights, including zero. The referee also remarked that our methods have a similar flavor to Tauberian theorems (see, for example, [5]). In particular, our proof of Theorem 3 relies on the asymptotic for $E_{k}(q)$ as $q \rightarrow 1$, which we deduce via modularity.

The first author was supported by a post-doctoral fellowship from the Natural Sciences and Engineering Research Council of Canada (NSERC). The second author was supported by an NSERC Discovery Grant.

\section{References}

[1] J. H. Bruinier and K. Ono, Algebraic formulas for the coefficients of half-integral weight harmonic weak Maass forms, preprint (2011).

[2] M. Dewar and M. R. Murty, A derivation of the Hardy-Ramanujan formula from an arithmetic formula, to appear in Proc. Amer. Math. Soc.

[3] W. Fulks, Advanced Calculus: An Introduction to Analysis (Wiley, 1964).

[4] G. H. Hardy and S. Ramanujan, Asymptotic formulae in combinatory analysis, Proc. London Math. Soc. (2) 17 (1918) 75-115.

[5] A. E. Ingham, A Tauberian theorem for partitions, Ann. of Math. (2) 42 (1941) 10751090.

[6] J. Lehner, Discontinuous Groups and Automorphic Functions, Mathematical Surveys, No. 8 (American Mathematical Society, Providence, RI, 1964).

[7] H. Petersson, Über die Entwicklungskoeffizienten der automorphen Formen, Acta Math. 58(1) (1932) 169-215.

[8] H. Rademacher, The Fourier coefficients of the modular invariant $j(\tau)$, Amer. J. Math. 60(2) (1938) 501-512. 